\section{Multi-drug resistant Helcococcus-like organism isolated from a chest abscess}

\author{
Isabella Martin, ${ }^{1}$ Joseph Schwartzman, ${ }^{1}$ \\ Kathryn Ruoff ${ }^{2}$ \\ 'Department of Pathology, Dartmouth- \\ Hitchcock Medical Center, Lebanon, NH; \\ 2Department of Microbiology and \\ Immunology, Dartmouth College, \\ Hanover, NH, USA
}

\begin{abstract}
We report a case of multi-drug resistant Helcococcus-like organism isolated in pure culture from a chest abscess in a 31-year-old pregnant woman.
\end{abstract}

\section{Case Report}

A 31-year-old pregnant female at 27 weeks gestation was referred to DartmouthHitchcock Medical Center's dermatology clinic (USA) in August 2009 for evaluation of an abscess on the chest associated with an epidermal inclusion cyst. She had a history of a prior abscess being drained in the same area in December 2005. Her medical/surgical history included cholecystectomy (1999), dilation and curettage for miscarriage (2008) and left knee surgery (2005).

Physical examination revealed a firm, mobile subcutaneous nodule with overlying punctum on the right medial chest. There was a superimposed fluctuant pustule with surrounding erythema. The patient had no systemic symptoms. Incision and drainage (I\&D) was performed. A significant amount of purulent fluid was drained and a swab specimen was sent for gram stain and culture. No surgical pathology specimen was submitted. The patient was sent home with a prescription for cephalexin $500 \mathrm{mg}$ three times a day for five days.

The gram stain showed moderate polymorphonuclear leukocytes and moderate gram positive cocci in clusters. The specimen was inoculated onto trypticase soy agar containing 5\% sheep blood (TSA), MacConkey agar, chocolate agar, and Columbia agar with 5\% sheep blood (CNA) (Remel, Lenexa, KS, USA). The TSA and chocolate agars were incubated at $35^{\circ} \mathrm{C}$ in a $\mathrm{CO}_{2}$-enriched (5\%) atmosphere, while the MacConkey and CNA agars were incubated aerobically at $35^{\circ} \mathrm{C}$. No growth was seen at 24 hours. At 48 hours, pinpoint, grey, non-hemolytic colonies were observed on chocolate agar. Gram stain of these colonies revealed gram positive cocci in clusters. Growth was not enhanced by anaerobic conditions. The organism was negative for catalase, pyrrolidonyl arylamidase (PYR) and esculin hydrolysis and its growth on chocolate agar suggested that it was not lipophilic.

An API 20 Strep system test (bioMérieux, Durham, NC, USA) was performed and read at 24 hours which showed weak positivity for hippurate and strong positivity for beta-galactosidase and leucine arylamidase. The organism was negative for acetoin production, beta-glucosidase hydrolysis, PYR, alpha-galactosidase, beta-glucuronidase, alkaline phosphatase, and arginine dihydrolase. The organism did not acidify ribose, arabinose, mannitol, sorbitol, lactose, trehalose, inulin, raffinose, starch or glycogen. These biochemical properties failed to result in species identification in the commercial kit database.

After 72 hours incubation, the isolate was identified further by $16 \mathrm{~S}$ rRNA gene sequencing. Nucleic acid was prepared with PrepMan Ultra reagent (Applied Biosystems, Foster City, CA, USA) and PCR was performed with a MicroSeq 500 kit (Applied Biosystems, USA) according to the manufacturer's instructions. ExoSAP-IT (USB Co., Cleveland, OH, USA) was used to purify the PCR product, which was sequenced at the Dartmouth College Molecular Biology and Proteomic Core Facility. The PCR product was sequenced twice, once with a forward primer and once with a reverse primer. The results of the forward and reverse primergenerated sequences were aligned and used to generate a consensus sequence. Two out of three unknown bases were identified through this alignment, leaving one unknown base. The National Center for Biotechnology Information (NCBI) GenBank BLAST program was then used to identify homologous sequences for 426 ribosomal RNA bases.

The genetic sequence of our isolate displayed $99 \%$ similarity to the sequence from the initially described $H$. sueciensis isolate (CCUG 47334 accession number NR 025606.1). Four hundred twenty-four of 426 bases were in alignment. Of the two base pairs not in alignment, one was an unknown base in the query strain while the other was an unknown base in the subject strain.

Antibiotic susceptibility was assessed using the E-Test method (AB Biodisk, Piscatawy, NJ, USA) on chocolate-agar with a number 0.5 McFarland standard-adjusted suspension of helcococci. The plates incubated for 24 hours at $35^{\circ} \mathrm{C}$ in an atmosphere containing $5 \% \mathrm{CO}_{2}$. Testing was performed for vancomycin, penicillin G, trimethoprim-sulfamethoxazole, ciprofloxacin and ceftriaxone. Results were expressed as susceptible, intermediate, or resistant according to the Clinical and Laboratory Standards Institute criteria for
Correspondence: Isabella Wetherill Martin, Department of Pathology, One Medical Center Drive, Lebanon, NH 03766, USA.

Tel.: +1.603.650.4809 - Fax: +1.603.650.4845

E-mail: isabella.w.martin@hitchcock.org

Key words: Helcococcus, antibiotics, resistance.

Acknowledgements: authors would thank Elissa Eckhardt, MT, Megan Read, MT, MPH and Nisalda Carreiro, MT.

Contributions: IM, drafting of manuscript, acquisition of data, final approval of the manuscript to be published; JS, revision of manuscript for important intellectual content, final approval of the manuscript to be published; KR, initial concept and design of case report, acquisition of data, revision of manuscript for important intellectual content, final approval of the manuscript to be published.

Conflict of interest: the authors declare no potential conflict of interest.

Conference presentation: this case report was presented in poster form at the College of American Pathologists annual meeting in Chicago, IL, USA, September, 2014. No funding was used to support this case report.

Received for publication: 9 December 2014. Revision received: 5 February 2015.

Accepted for publication: 5 February 2015 .

This work is licensed under a Creative Commons Attribution NonCommercial 3.0 License (CC BYNC 3.0).

(O) Copyright I. Martin et al., 2015

Licensee PAGEPress, Italy

Infectious Disease Reports 2015; 7:5754

doi:10.4081/idr.2015.5754

gram positive cocci. ${ }^{1}$ Vancomycin showed a minimum inhibitory concentration of 4 micrograms/mL (susceptible range $\leq 1$ microgram /mL for Streptococcus). No zone of inhibition was observed for any of the other antibiotics tested [resistance range defined as follows according to package inserts: $\geq 4$ for penicillin G $(S$. viridans $), \geq 4$ for trimethoprim/sulfamethoxazole (aerobes), $\geq 4$ for ciprofloxacin (aerobes), $\geq 4$ for ceftriaxone (Viridans group)]

Upon telephone follow-up three days after I\&D, the patient reported much improvement and resolution of pain and redness.

\section{Discussion}

Helcococcus is a genus of facultatively aerobic, gram positive, catalase negative cocci first described in 1993 with a collection of 9 strains 
from human clinical specimens. ${ }^{2}$ Initially thought to be a commensal organism of the lower extremity with potential to function as an opportunistic pathogen in immunocompromised patients, ${ }^{3}$ it has since been described as a pure isolate from infections in immune-competent patients. ${ }^{4-6}$ In the 20 years since its first description, at least 20 cases have been reported in the literature, most of which have been H. kunzii. Clinical sources of specimens predominantly have been wounds of the lower extremities, although cases have also been reported from the breast (described alternately as masses and abscesses), ${ }^{2,4}$ face, ${ }^{3}$ prosthetic joints ${ }^{6}$ cardiac valves, ${ }^{7}$ an implantable cardiac device $^{8}$ and a brain abscess. ${ }^{9}$ No cases in pregnant women have been reported to date.

In 2004, a novel species of the genus was reported from the wound of an 83-year old Swedish woman and given the name Helcococcus sueciensis. ${ }^{10}$ This initial case (bacterial isolate CCUG 47334) was described and classified using biochemical, molecular chemical and molecular phylogenetic evidence. Specifically, API Rapid 32 Strep system and API Rapid ID 32A System were used to assess biochemical properties while determination of DNA G + C content of $29.5 \mathrm{~mol} \%$ and whole-cell protein profiling were performed to analyze the molecular chemical and molecular phylogenetic properties respectively. No antibioticsusceptibility testing results were reported for this species. Two additional isolates of $H$. sueciensis have recently been described.

While the $16 \mathrm{~S}$ results for our isolate point toward an identification of $H$. sueciensis, the biochemical profile differs significantly from the first described strain. Additionally, unlike the initially-described strain, the growth of our specimen was not enhanced in an anaerobic environment. It is also possible that our isolate represents a new species of Helcococcus. While we are confident that our species belongs to the Helcococcus genus, definitive identification to the species level is not possible based on our data. Additional descriptions of Helcococcus isolates will provide a more robust framework from which to categorize distinct species.

Our isolate was found to be multi-drug resistant, a characteristic only recently described. Standardized susceptibility testing methods have not been established for Helcococcus spp. Of the fifteen published articles that describe Helcococcus infections in humans, twelve contain information about antibiotic susceptibility based on E-test, diskdiffusion, Microscan or Sensititre systems using the breakpoints recommended by the National Committee for Clinical Laboratory Standards (CLSI) for gram positive cocci. ${ }^{1}$ Of these articles, five describe strains that are susceptible to all antibiotics tested, two describe strains with vancomycin resistance, ${ }^{7,11}$ one describes a range of resistance to intermediate susceptibility in $10 \mathrm{H}$. kunzii isolates to ciprofloxacin ${ }^{3}$ (MIC ranging from 2 to 4 micrograms/mL), and three describe erythromycin resistance ${ }^{12}$ one in combination with aminoglycoside resistance ${ }^{13}$ and another in combination clindamycin resistance. ${ }^{4}$ In contrast to these reports of single or double antibiotic resistance, a recent study describing five Helcococcus isolates (2 kunzii, 2 sueciensis and 1 novel strain named seattlensis) found intermediate to high MICs for sulfamethoxazole-trimethoprim, gentamicin, erythromycin, azithromycin, clindamycin, moxifloxacin, and levofloxacin. ${ }^{14}$ Similarly, our organism was resistant to all five antibiotics tested. However, given the superficial nature of the patient's cyst, incision and drainage proved to be adequate therapy to resolve her infection and antibiotics were not necessary.

\section{Conclusions}

With this report we add to the helcococcal literature a case of epidermal inclusion cyst infected with a multi-drug resistant Helcococcus-like species from the chest in a 31-year old pregnant woman. Future antibiotic resistance in helcococcal organisms could raise treatment challenges.

\section{References}

1. Clinical and Laboratory Standards Institute. Performance standards for antimicrobial susceptibility testing; twenty-fourth informational supplement. CLSI document M100-S24. 2014.

2. Collins MD, Facklam RR, Rodrigues UM, Ruoff KL. Phylogenetic analysis of some Aerococcus-like organisms from clinical sources: description of Helcococcus kunzii gen. nov., sp. nov. Int J Syst Bacteriol 1993;43:425-9.

3. Caliendo AM, Jordan CD, Ruoff KL. Helcococcus, a new genus of catalase-negative, gram-positive cocci isolated from clinical specimens. J Clin Microbiol 1995; 33:1638.

4. Chagla AH, Borczyk AA, Facklam RR, Lovgren M. Breast abscess associated with Helcococcus kunzii. J Clin Microbiol 1998;36:2377-9.

5. Peel MM, Davis JM, Griffin KJ, Freedman DL. Helcococcus kunzii as sole isolate from an infected sebaceous cyst. J Clin Microbiol 1997;35:328-9.

6. Perez-Jorge C, Cordero J, Marin M, Esteban J. Prosthetic joint infection caused by Helcococcus kunzii. J Clin Microbiol 2012;50:528-30.

7. Fitzgerald SF, Crowe MJ, Cassidy B, et al. A novel Helcococcus-like organism causing endocarditis in an injecting drug user. $\mathrm{J}$ Heart Valve Dis 2005;14:693-4.

8. McNicholas S, McAdam B, Flynn M, Humphreys $H$. The challenges of implantable cardiac device infection due to Helcococcus kunzii. J Hosp Infect 2011;78:337-8.

9. Sridhar S, Chan JF, Yuen KY. First report of brain abscess caused by a satelliting phenotypic variant of Helcococcus kunzii. J Clin Microbiol 2014;52:370-3.

10. Collins MD, Falsen E, Brownlee K, Lawson PA. Helcococcus sueciensis sp. nov., isolated from a human wound. Int J Syst Evol Microbiol 2004;54:1557-60.

11. Panackal AA, Houze YB, Prentice J, et al. Prosthetic joint infection due to Helcococcus pyogenes. J Clin Microbiol 2004;42:2872-4.

12. Woo PC, Tse H, Wong SS, et al. Life-threatening invasive Helcococcus kunzii infections in intravenous-drug users and ermAmediated erythromycin resistance. J Clin Microbiol 2005;43:6205-8.

13. Riegel P, Lepargneur JP. Isolation of Helcococcus kunzii from a post-surgical foot abscess. Int J Med Microbiol 2003;293: 437-9.

14. Chow SK, Clarridge JE 3rd. Identification and clinical significance of Helcococcus species with description of Helcococcus seattlensis sp. nov. from a patient with urosepsis. J Clin Microbiol 2014;52:854-8. 